Nervenarzt 2020 $91: 422-432$

https://doi.org/10.1007/s00115-020-00894-0

Online publiziert: 27. März 2020

(c) Der/die Autor(en) 2020

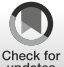

\author{
S. Seidenbecher ${ }^{1,2} \cdot$ C. Steinmetz $\cdot$ A.-M. Möller-Leimkühler ${ }^{3}$ B. Bogerts ${ }^{1,2,4}$ \\ 'Salus Institut, Salus gGmbH, Magdeburg, Deutschland \\ ${ }^{2}$ Klinik für Psychiatrie und Psychotherapie, Otto-von-Guericke-Universität Magdeburg, Magdeburg, \\ Deutschland \\ ${ }^{3}$ Klinik für Psychiatrie und Psychotherapie, Ludwig-Maximilians-Universität München, München, \\ Deutschland \\ ${ }^{4}$ Center of Behavioral Brain Sciences (CBBS), Magdeburg, Magdeburg, Deutschland
}

\title{
Terrorismus aus psychiatrischer Sicht
}

\section{Hintergrund und Fragestellung}

Laut Global Terrorism Index [38] starben im Jahr 2018 bei Terroranschlägen weltweit 15.952 Menschen. Nach einem stetigen Ansteigen des weltweiten Terrorismus mit Höchstwerten im Jahr 2014 (33.555 Todesfälle) ist seit der sich abzeichnenden Niederlage des sog. Islamischen Staates (IS) weltweit ein Rückgang von Terroranschlägen zu verzeichnen. Auch in Europa sanken die Todesfälle seit 2016 um $75 \%$, wobei für Westeuropa die niedrigste Zahl an Vorfällen seit 2012 berichtet wird. Dennoch ist die Zahl der Opfer von Terroranschlägen heute noch dreimal so hoch wie eine Dekade zuvor. Zudem hat in Westeuropa, Nordamerika und Australien im dritten Jahr in Folge der rechtsextreme Terrorismus zugenommen (Anstieg zwischen 2014 und 2018 um $320 \%)$.

Die politischen, gesellschaftlichen und psychosozialen Konstellationen sind für viele Einwohner einer vom Terror betroffenen Region relevant, jedoch wird nur ein äußerst geringer Anteil von ihnen zu Terroristen. Ziel der vorliegenden Arbeit ist es, neben einer kurzen Darstellung historischer Aspekte und verschiedener Formen von Terror, aus der zu dieser Thematik verfügbaren Literatur eine Übersicht über das multifaktorielle Bedingungsgefüge mit besonderer $\mathrm{Be}$ achtung psychopathologischer Aspekte von Menschen, die Terrorhandlungen durchführen, zusammenzustellen. Neben der Sichtung älterer Literatur zur RAF in den 1970er- und 1980er-Jahren erfolgte eine Literatursuche über MEDLINE/PubMED, SCOPUS, PsychInfo und PsychARTICLES für den Zeitraum 2000 bis 2019 unter den Schlüsselworten „terrorism“, „terrorists“, „psychology“, "psychopathology“ bzw. „psychiatry“. Eingeschlossen wurden nur Publikationen, in denen empirische Daten zur Psychologie bzw. Psychopathologie oder $\mathrm{zu}$ sozialen Bedingungsfaktoren von Terroristen enthalten waren.

\section{Begriffsbestimmung}

Der Begriff „Terrorismus“ ist ein heterogenes Konstrukt, zu dessen Definition es keinen Konsens gibt. Entsprechend unscharf und variabel wird dieser Begriff in politischen Kontexten verwendet. Abzugrenzen ist Terrorismus von Amok (persönliche Motive, häufig im engen sozialen Umfeld, hohe Prävalenz psychischer Vorerkrankungen [10]), „school shootings“ (Amokläufe an Schulen), Aktionen von Guerillas oder paramilitärischen Organisationen sowie organisierter Kriminalität. Um per definitionem von einem terroristischen Anschlag sprechen zu können, muss es sich bei der Attacke um einen absichtlichen Gewaltakt mit politischen oder ideologischen Motiven handeln, dessen Ziel häufig ein Symbolwert für ein verhasstes System zukommt.

\section{Historischer Hintergrund und aktuelle Entwicklungen}

Terrorismus ist ein weltweites und keinesfalls neues Phänomen. Bekannte his- torische Beispiele sind die jüdischen Widerstandskämpfer gegen die Römer im 7. Jahrhundert oder die Selbstmordanschläge islamistischer Assassinen gegen politische Widersacher oder Ungläubige im 11. Jahrhundert. Terroristische Aktionen bleiben bis heute ein in der Historie stets präsentes Phänomen extremer politisch oder ideologisch motivierter Gewalthandlungen [52].

Rapoport [69] beschreibt eine zeitliche Abfolge des Terrorismus der letzten 200 Jahre in vier Perioden. Die erste Phase, welche sich vom 19. Jahrhundert bis zum Ersten Weltkrieg erstreckt, bezeichnet er als anarchistische Welle. Anschließend, beginnend in den 1920erJahren, entwickelte sich eine Phase antikolonialer Terrorakte motiviert durch ethnisch-nationalistische Einstellungen zu Zeiten der Unabhängigkeits- und Widerstandsbewegungen. Im Zeitraum der 1960er- bis 1980er-Jahre entstand die dritte, überwiegend linksradikal geprägte Terrorwelle. Die „Rote Armee Fraktion“ (RAF) verfolgte zu dieser Zeit in Deutschland das Ziel, durch Terroranschläge auf Repräsentanten von Staat und Gesellschaft das von ihr zum Feindbild erklärte kapitalistische System zu Fall $\mathrm{zu}$ bringen. In diesem Zusammenhang ist auch das Sozialistische Patientenkollektiv zu erwähnen, das vorgab, für die „Abschaffung der krankmachenden privatwirtschaftlich-patriarchalischen Gesellschaft“ [23] kämpfen zu müssen. Einige der Psychiatriepatienten dieses Kollektivs schlossen sich später der RAF an und waren unter anderem an dem 
Anschlag auf die Deutsche Botschaft in Stockholm im April 1975 beteiligt. Weitere Beispiele dieser dritten Terrorwelle sind die Rote Brigade in Italien sowie die Nihon Sekigun (Japanische RAF). Daran anschließend und bis heute andauernd beschreibt Rapoport eine vierte Terrorwelle, welche vornehmlich von islamistisch-dschihadistischen Motiven getragen wird [69].

Als weitere Terrorwelle gewann in den letzten zehn Jahren der Rechtsterrorismus an Bedeutung. Rechtsradikaler Terrorismus war Gegenstand des im Juli 2018 vor dem Oberlandesgericht München abgeschlossenen Prozesses gegen die rechtsextreme Terrorgruppe Nationalsozialistischer Untergrund (NSU), die zwischen 2000 und 2007 zehn rassistisch motivierte Morde, 43 Mordversuche, drei Sprengstoffanschläge und 15 Raubüberfälle verübte. Im Rahmen der Flüchtlingsbewegung nahmen rechtsextremistische Taten in Deutschland zu, wie z.B. die Anschläge auf Asylantenheime in Rostock-Lichtenhagen 1992 und in Freital 2015/2016. Fremdenfeindliche Hintergründe hatten auch die Anschläge auf die Oberbürgermeisterin in Köln (Oktober 2015), den Regierungspräsidenten in Kassel (Juni 2019), die Synagoge in Halle (Oktober 2019) und auf Menschen mit Migrationshintergrund in München (Juli 2016) sowie Hanau (Februar 2020; [82]).

Eine neue Entwicklung ist der zunehmende Einsatz des Internets für terroristische Radikalisierungen und Aktionen. Dabei ist zu beobachten, dass auch Einzeltäter zunehmend in ein virtuelles extremistisches soziales Umfeld über Hassforen in Internetgruppen eingebunden sind und dadurch die Trennung zwischen Einzel- und Gruppentätern im digitalen Raum verschwimmt. Einer Studie des National Consortium for the Study of Terrorism and Responses to Terrorism (START) zufolge, welche das Social-media-Verhalten 479 US-amerikanischer Terroristen verfolgte, sind es vor allem Einzeltäter $(68,12 \%)$, welche sich über soziale Medien radikalisieren und mobilisieren [40]. Spätestens der Christchurch Anschlag im März 2019 mit 50 Toten und ebenso vielen Verletzten stellt in diesem Zusammenhang eine neue Kategorie eines hauptsächlich im Internet entstandenen internationalen rechten Terrors dar. In die gleiche Richtung sind die Anschläge von Poway (Kalifornien, April 2019), El-Paso (Texas, Juni 2019), Baerum (Norwegen, August 2019), Halle an der Saale (Oktober 2019) und Hanau (Februar 2020) einzuordnen [44, 51]. All diese Täter zitieren sich in Internetbotschaften gegenseitig und berufen sich ebenso, wie zuvor der norwegische Massenmörder Anders Behring Breivik (Utoya, Norwegen, Juli 2011), auf verschiedene Variationen von Verschwörungs- oder Verdrängungstheorien gegen die eigene Rasse [19].

In das multifaktorielle Bedingungsgefüge terroristischer Gewalt fließen biografische, psychopathologische und psychosoziale Faktoren ein, auf welche im Folgenden näher eingegangen wird.

\section{Psychosoziales und psycho- pathologisches Bedingungs- gefüge von Gruppenterror}

Oft wird die Auffassung vertreten, dass Terrorhandlungen nicht von psychisch gesunden Menschen verübt werden können, sondern vielmehr eine Persönlichkeitsstörung, wenn nicht sogar eine affektive, psychotische oder posttraumatische Erkrankung vorliegen müsste [85], die in einer Bereitschaft zur Radikalisierung bzw. Neigung zu Gewalttaten resultiere [7]. Gleichwohl stellen mentale Erkrankungen nur einen nachgeordneten Faktor im Bedingungsgefüge von Terrorhandlungen dar [28, 52]. Zudem ist hervorzuheben, dass die weit überwiegende Mehrheit von Menschen mit psychischen Störungen keine kriminellen Handlungen begeht. Gewaltfrei leben $95 \%$ der psychisch Erkrankten im Vergleich zu $98 \%$ der Menschen ohne psychische Störungen [50].

\section{Untersuchungen zum Linksterrorismus}

Ausführlichere Untersuchungen zu den psychologischen, sozialen und psychopathologischen Bedingungsfaktoren terroristischer Handlungen erfolgten erstmals vor etwa vier Jahrzehnten anlässlich des damals in der Bundesrepublik dominierenden RAF-Terrors [39, 70, 76, 83]. Vor diesem Hintergrund erstellte Schmidtchen [76] anhand von Fahndungsunterlagen und Prozessakten eine Zusammenfassung der biografischen Daten von 23 rechtsextremistischen und 227 linksextremistischen Personen. Letztere waren überwiegend der RAF und der Bewegung 2. Juni zuzuordnen, hatten ein überdurchschnittliches gesellschaftliches Herkunftsniveau und zum großen Teil ein Hochschulstudium mit oder ohne Abschluss. Ein Drittel der Mitglieder linksterroristischer Gruppierungen waren Frauen. Dagegen wurde Rechtsterrorismus ausschließlich durch männerbündnerische Gruppen mit ausgeprägtem Uniform-, Waffenund Kampfkult praktiziert bei wesentlich geringerem Durchschnittsalter (22 vs. 30 Jahre). Ein Drittel aller Terroristen fiel schon vor Anschluss an die Terrorszene durch Jugendstraftaten auf [76].

Süllwold [83] untersuchte psychologische Aspekte anhand biografischer Daten von 273 Linksterroristen. Der größte Anteil an Studenten mit späterer terroristischer Karriere bewegte sich in ideologisch homogenen Zirkeln mit starkem Konformitätsdruck und lebte in kollektiven Wohnformen wie Kommunen. Gruppenzugehörigkeit wurde zur psychischen Existenzfrage. Ein Drittel der Linksextremisten hatte einen schwerwiegenden irreparablen Bruch mit dem Elternhaus. Gewalt wurde als Gegengewalt zur imperialistischen Politik legitimiert. Jedoch habe nicht die Zielsetzung, sondern die terroristische Destruktion selbst die eigentliche Befriedigung verschafft. Psychische Störungen (entsprechend Achse I, DSMIV) waren bei Terroristen nicht häufiger als in der Durchschnittsbevölkerung vorhanden. Oft aber seien besonders ausgeprägte Persönlichkeitszüge, wie „extreme Extroversion“ und "neurotische Feindseligkeit", festzustellen (am ehesten histrionische und paranoide Persönlichkeitsakzentuierungen). Terroristen gemeinsam sei ein unerfüllter Geltungs- und Machtanspruch junger Menschen, die die Gesellschaft durch Gewaltmittel zwingen wollten, von ihrer 
Nervenarzt 2020 $91: 422-432$ https://doi.org/10.1007/s00115-020-00894-0

(c) Der/die Autor(en) 2020

\section{S. Seidenbecher · C. Steinmetz · A.-M. Möller-Leimkühler · B. Bogerts}

\section{Terrorismus aus psychiatrischer Sicht}

\section{Zusammenfassung}

Hintergrund. Terrorismus gehört zu den Extremformen von Gewalt, die bisher in der deutschsprachigen psychiatrischen Fachliteratur kaum Beachtung fanden. Nach Terroranschlägen oder Attentaten kommt jedoch regelhaft die Frage auf, ob die Mentalität der Täter noch im Bereich psychischer Normalität liegt.

Fragestellung und Methode. Ziel dieser Übersichtsarbeit ist es, das multidimensionale Ursachengefüge von Terrorismus mit besonderer Beachtung psychopathologischer Gegebenheiten bei den Tätern darzustellen. Hierzu wurde neben einer kurzen Zusammenfassung des historischen Hintergrundes eine Literatursuche in PubMed, SCOPUS, Psychlnfo, und PsychARTICLES durchgeführt.

Ergebnisse. Aus psychiatrischer Sicht ist eine Unterscheidung von Einzel- und Gruppentätern essenziell, wobei erstere eine deutlich höhere Prävalenz an krankheitswertigen Psychosyndromen, insbesondere an psychotischen, anhaltenden wahnhaften, aber auch affektiven Störungen, aufweisen. Bei der Mehrzahl der untersuchten terroristischen Gruppentäter konnten keine derartigen psychiatrischen Diagnosen (nach Achse I, DSM-IV) festgestellt werden. Bei diesen Tätern spielen neben biografischen, Sozialisierungs-, gruppendynamischen sowie ideologischen Aspekten Persönlichkeitsakzentuierungen bis hin zu Persönlichkeitsstörungen (vor allem narzisstischer und paranoid-fanatischer Prägung) eine Rolle. Es wird dargelegt, warum Terrorismus ein vorwiegend männliches Phänomen ist.

Schlussfolgerungen. Es liegt ein komplex interagierendes Bedingungsgefüge aus biographischen, biologischen, psychopathologischen, soziologischen und ideologischen
Komponenten vor, das bei Einzeltätern und Gruppenterroristen unterschiedlich gewichtet ist. Die Ursachen für Terrorhandlungen sind weniger in gewaltaffinen Ideologien selbst zu suchen als darin, dass Individuen mit vorbestehender hoher Gewaltaffinität, sei sie persönlichkeitsinhärent oder biografisch herleitbar, sich Ideologien anschließen, mit denen sich terroristische Gewaltakte rechtfertigen lassen. Die Möglichkeiten der Psychiatrie in Früherkennung und Prävention sind begrenzt. Auf neu entwickelte Skalen zur Beurteilung eines Risikos für extreme Gewalttaten wird hingewiesen.

\section{Schlüsselwörter}

Terror · Psychopathologie - Einzeltäter · Terroristische Gruppierungen · Gewalt

\section{Psychiatric aspects of terrorism}

\section{Abstract}

Background. Terrorism belongs to the extreme forms of violence that have so far received little attention in psychiatric research and are rarely mentioned in textbooks of psychiatry. After terror attacks, however, the question regularly arises whether terrorists suffer from mental disorders.

Objective and methods. The aim of this review is to summarize the multidimensional causes of terrorism with special emphasis on psychopathological aspects of the perpetrators. In addition to a brief summary of the historical background and recent developments in terrorism, a literature search was performed using PubMed, SCOPUS, PsychInfo and PsychARTICLES.
Results. From a psychiatric point of view, a differentiation between lone terrorists and group terrorists is essential. Lone terrorists have a much higher prevalence of psychiatric disorders, such as psychotic, paranoid and affective symptoms. The majority of terrorists acting in groups rarely suffer from such mental disorders. For these perpetrators biographic aspects and socialization, group dynamics and ideological personality profiles with narcissistic, histrionic, fanatic and antisocial components are more relevant. The phenomenon of terrorism predominantly being a male domain is discussed.

Conclusion. The manifold manifestations of terrorism are caused by complex patterns of interacting biographic, sociological, ideological and psychopathological components that differ between lone acting and group terrorists. The real causes for acts of terrorism are not various ideologies permitting violence but consist more of a pre-existing violenceoriented mentality of the perpetrators looking for such ideologies to justify their acts. The possibilities of psychiatry in prevention and early recognition are limited. Some recently developed scales for risk assessment of extreme violence are mentioned.

\section{Keywords}

Terror · Psychopathology · Lone acting terrorists · Terrorist groups · Violence
Person und ihrem Willen Kenntnis zu nehmen [83].

Jäger und Böllinger [39] kamen zu der Auffassung, Terrorismus enthalte einen hohen Anteil normalen Sozialverhaltens, das nur verständlich werde, wenn man terroristische Gruppen als Subkulturen mit eigenem Wertesystem betrachte. Für eine psychopathologische Interpretation terroristischen Handelns gebe es keine Anhaltspunkte. Nicht auszuschließen sei jedoch, dass es psychopathologische Fak- toren in einzelnen Individualentwicklungen gebe. Für eine generelle Pathologisierung von Terroristen fehle jeglicher Anhaltspunkt [39].

Dieselbe Auffassung vertrat Rasch [70], der elf angeklagte Terroristen forensisch-psychiatrisch begutachtete und keine Anhaltspunkte für krankheitswertige psychische Störungen finden konnte. Dies treffe auch für die Hauptfiguren des Stuttgart-Stammheim-Prozesses, Baader, Meinhof, Enslin und Raspe, zu.
Er vertrat die Meinung, dass die terroristischen Entwicklungen am ehesten mithilfe der Frustrations-AggressionsTheorie (Bandura) als Reaktion auf die damaligen politischen Verhältnisse in Westdeutschland und durch gruppendynamische Prozesse innerhalb der RAF zu erklären seien [70]. 


\section{Untersuchungen zum islamistischen Terror}

Der mit dem Anschlag auf das World Trade Center am 11.09.2001 beginnende weltweite islamistische Terror hatte eine neue Publikationswelle zur Frage der Ursachen von Terror zur Folge. Die für die linksterroristische Szene der RAFZeit getroffene Feststellung, wonach bei Gruppenterroristen keine erhöhte Inzidenz krankheitswertiger Psychosyndrome vorlag, wurde in ähnlicher Weise auch in den neueren Untersuchungen zum islamistischen Terror getroffen. Sageman [74] stellte fest, dass mehr als $70 \%$ islamistischer Terroristen eine College-Ausbildung hatten und über $50 \%$ eine Berufsausbildung absolvierten. Er fand keine Hinweise auf krankheitswertige Persönlichkeitsstörungen oder paranoide Züge.

Bartletts und Miller [6] verglichen Persönlichkeitsprofile von 61 gewalttätigen islamistischen Terroristen mit 28 islamistisch radikalisierten Personen ohne Gewaltneigung sowie 70 jungen Muslimen ohne Radikalisierungstendenzen in Kanada, Dänemark, England, Frankreich und den Niederlanden. Unter den Radikalisierten waren Personen mit technischen oder akademischen Abschlüssen überrepräsentiert. Gefühlte oder tatsächliche Diskriminierung oder die Auffassung, dass der Islam von der westlichen Welt bedroht werde und deshalb verteidigt werden müsse, wurden als häufigste Motive für terroristische Handlungen genannt. Bemerkenswert ist, dass die weit überwiegende Mehrzahl der Terroristen weder eine religiöse Erziehung noch profundere Kenntnisse des Korans hatte. Als wichtigste Differenz zwischen Radikalisierten, die nicht gewalttätig wurden, und solchen, die gewalttätig wurden, wurde bei Letzteren eine emotionale Disposition zu gewalttätigem Verhalten, Stimulation und „Kick“ durch Gewaltakte, ein terroristischer Ehrenkodex bei ausgeprägter „Wir-gegenSie“-Narrative sowie Gruppendruck angesehen. Psychische Störungen wurden nicht weiter thematisiert [6].

Merari [57] verglich 15 Selbstmordattentäter mit 14 Terroristen, die sich nicht suizidierten. Die Suizid-Terroristen hat- ten einen höheren Ausbildungsstand und bessere wirtschaftliche Verhältnisse sowie eine tiefere Religiosität bei gehäuftem Vorkommen vermeidender, dependenter und depressiver Persönlichkeitszüge.

Leygraf [47] erstattete über 29 islamistisch motivierte Straftäter forensischpsychiatrische Gutachten. Davon waren 19 eingewanderte Täter, bei denen er keine psychopathologischen Symptome fand, jedoch dissoziale Auffälligkeiten. Von den zehn in Deutschland aufgewachsenen Islamisten waren drei an einer schizophrenen Psychose erkrankt, zwei hatten eine primär dissoziale Problematik.

Leuschner et al. [46] berichten, dass weniger eine krankheitswertige Psychopathologie als Persönlichkeitsakzentuierungen im Übergangsbereich zu Persönlichkeitsstörungen im Zusammenhang mit terroristischer Radikalisierung von Terrorgruppen $\mathrm{zu}$ sehen sind. Auffällig seien narzisstische und „psychopathische“ Persönlichkeitsstrukturen (im Sinne von R. Hare [33]) sowie ein generell erhöhtes Aggressionspotenzial. Post beschreibt in einer Übersichtsarbeit [65] ebenso wie Bhui et al. [8] das in Terrorgruppen gehäufte Vorliegen narzisstischer und antisozialer Persönlichkeitsprofile. Terroristen würden nicht wahrscheinlicher an einer mentalen Erkrankung der Achse I nach DSM IV, wie z.B. einer psychotischen Störung, leiden als Menschen mit vergleichbarem sozialem Hintergrund [66]. Auch die Gruppe um Piccinni [64] argumentiert, dass keine Evidenz dafür bestehe, dass gruppenterroristisches Verhalten das Vorliegen einer psychiatrischen Erkrankung bzw. der Diagnose „Psychopathy“ zur Voraussetzung habe. Gleichfalls kommt Horgan [36] zu dem Schluss, dass es keine speziellen individuellen psychopathologischen Merkmale gebe, die Terroristen von der generellen Population unterscheiden.

\section{Kriminologische Analysen von IS-Zuläufern}

Gegen die Auffassung psychischer Unauffälligkeit von Personen, die sich Terrorgruppen anschließen, sprechen jedoch kriminologische Analysen des Bundesamtes für Verfassungsschutz.
Dieses legte 2016 eine Analyse der Radikalisierungshintergründe und -verläufe der Personen vor, die aus islamistischer Motivation aus Deutschland in Richtung des sog. Islamischer Staates (IS) nach Syrien oder in den Irak ausgereist waren [11]. Zwei Drittel der Ausgereisten waren schon vor ihrer Ausreise polizeibekannt, insbesondere wegen $\mathrm{Ge}$ walt- oder Eigentumsdelikten, darüber hinaus durch Straftaten aus dem Bereich „politisch motivierte Kriminalität“ sowie wegen Drogenhandels oder -besitzes. Im Verlauf der Radikalisierung war eine Zunahme politisch motivierter Kriminalität zu verzeichnen, gefolgt von Gewaltdelikten und Eigentumsdelikten. Mehr als die Hälfte dieses Personenkreises trat mit drei oder mehr Straftaten in Erscheinung. Es handelte sich somit überwiegend um Mehrfachtäter. Vorbestehende dissoziale Persönlichkeitszüge waren vielfach dokumentiert. Weitere Faktoren, die zur Radikalisierung beitrugen, waren Bekannte aus der Islamistenszene, Kontakte in einschlägigen Moscheen, Internet, Islamseminare und Koran-Verteilaktionen [11].

Vergleichbare Daten über Personen, die sich dem IS anschlossen, liegen aus Frankreich, England, Italien, Belgien, den Niederlanden und Griechenland vor [38]. Demnach hatten $50-70 \%$ bereits eine kriminelle Karriere vor Eintritt in die Terrorszene. Eine Erklärung für den hohen Anteil vorbestrafter Personen wurde darin gesehen, dass deren Neigung zu aggressiv-gewalttätigem Verhalten im terroristischen Umfeld besser ausgelebt werden kann und risikofreier ist.

Eher als das Vorliegen einer manifesten Psychopathologie psychotischer oder affektiver Art scheint bei Mitgliedern von Terrorgruppen eine besondere Persönlichkeitsakzentuierung bis hin zur Persönlichkeitsstörung vorzuliegen. Beschrieben werden narzisstische, antisoziale und paranoid-aggressive Persönlichkeitszüge [46].

\section{Unterschiedliche Radikalisierungs- wege}

Jensen et al. [41] untersuchten die Radikalisierungswege von 31 gewalttätigen im Vergleich zu 25 nichtgewalttätigen Extre- 
misten in den Vereinigten Staaten zwischen 1960 und 2013.90\% waren Männer im Alter von 28 bis 32 Jahren, mehr als die Hälfte hatte einen College-Abschluss. Die Wege, die zur gewalttätigen Radikalisierung führten, waren äußerst unterschiedlich. Persönliche und kollektive Krisen nach empfundener oder tatsächlicher Erniedrigung und Ausgrenzung verbunden mit kognitiver Neuausrichtung sowie neues Bedeutsamkeits-, Zugehörigkeits- und Verpflichtungsgefühl in der Terrorgruppe waren die wichtigsten Motive. Das Bedingungsgefüge zu gewalttätiger Radikalisierung erwies sich als äußerst komplex, einen einheitlichen Prototypen gab es nicht [41].

In einer Studie mit 227 links- und 23 rechtsextremen Terroristen konnte Crenshaw mittels eines semistrukturierten Interviews zwei Muster von Persönlichkeitseigenschaften differenzieren: Es handelt sich zum einen um einen extrovertierten, nach Anreiz suchenden und abhängigen Typus. Das zweite Muster wird als feindselig, grausam und abwertend beschrieben [18]. Eine Einteilung in Muster oder das Zuschreiben bestimmter Eigenschaften werden jedoch nicht dem Aspekt genüge, dass bei Terroristen ein breites Spektrum von Persönlichkeitsprofilen mit heterogenen psychologischen und psychopathologischen Charakteristika sowie ein komplexes Gefüge unterschiedlicher psychosozialer Erfahrungen vorliegen [64].

Webber und Kruglanski [86] formulierten drei wesentliche psychosoziale Voraussetzungen zur Entwicklung terroristischen Verhaltens: erstens eine individuelle Motivation, die aus einer Erniedrigung, Demütigung, Diskriminierung oder Unterdrückung resultieren könne, zweitens eine ideologische Narrative, mit der Gewaltanwendung gerechtfertigt werden könne und drittens ein soziales Netzwerk, das zur Durchführung radikaler Taten ansporne [86].

Bei terroristischen Tätern, die in Gruppen agieren, scheinen somit keine Hinweise auf erhöhte Prävalenzen manifester psychotischer oder affektiver Störungen vorzuliegen $[3,7,16]$. Es dominieren hingegen Persönlichkeitsprofile, die entweder gar nicht oder nur schwer in die gängigen Klassifi- kationssysteme nach ICD oder DSM einzuordnen sind und eine Nähe zu bestimmten Persönlichkeitsstörungen (Gruppe F6 nach ICD-10 bzw. AchseII-Störungen nach DSM-IV) aufweisen.

\section{Sonderfall salafistischer Terrorismus}

Salafismus ist per se nicht mit Extremismus oder Terrorismus gleichzusetzen. Salafisten beanspruchen den wahren Islam, der sich auf die Gründergeneration bezieht, nur für sich und sehen Angehörige anderer Religionen oder Atheisten als „Ungläubige“ an. Bei gewaltorientierten Gruppen kann dies Tötungsaufrufe nach sich ziehen, insbesondere, da extremistische Salafisten den Islam als bedroht ansehen. Deshalb werde der Dschihad, der Heilige Krieg, notwendig. Dschihadisten nehmen den eigenen Tod in Kauf, um - wie sie glauben - den Islam zu verteidigen. Dafür wird der direkte Einzug in das Paradies verheißen.

Überlebende Attentäter berichteten, dass die Sehnsucht nach einem Heldentod und Unsterblichkeit eine starke Motivation ist. Andere sprachen von der Ekstase des Kampfes, die an religiöse Begeisterung heranreichte [74]. Bei einem der Terroristen von 9/11 wurde ein Dokument mit Gebeten und Reflexionen gefunden, das den Attentätern auf das Word Trade Center helfen sollte, durchzuhalten.

\section{Untersuchung von terroristi- schen Einzeltätern}

Tatsächlich liegen psychische Erkrankungen vermehrt bei Personen vor, die als Einzeltäter, nicht aber im Rahmen einer Terrorgruppierung, agieren $[16,31]$. Alonso et al. konnten zeigen, dass insbesondere Individuen, die isoliert von Terrornetzwerken agieren, an psychischen Auffälligkeiten litten [3]. Demnach scheint aus psychiatrischer Sicht eine Unterscheidung der Täter in Einzeltäter (auch: „lone-wolves“, „leaderless resistance“, „freelance terrorism“ oder „individual terrorism“) und terroristische Gruppierungen („group-based terrorism“) sinnvoll zu sein $[16,78]$.
Einzeltäter können wie Gruppentäter einer bestimmten Ideologie folgen, die sie sich jedoch vorwiegend über das Internet angeeignet haben [1]. Gill und Kollegen postulieren, dass die sog. "lone-wolves“ nicht immer isoliert sind und es somit kein einheitliches Profil eines Einzeltäters gibt [30]. Vor allem in Bezug auf Einzeltäter lässt sich eine klare Dominanz des männlichen Geschlechts erkennen [45]. Außerdem sind Einzeltäter im Schnitt ca. zehn Jahre älter als Mitglieder terroristischer Organisationen [30]. Auch die Anzahl früherer Verurteilungen ist bei Einzeltätern höher [25].

Lindekilde et al. [48] untersuchen 33 terroristische Einzeltäter und unterteilten diese aufgrund des Persönlichkeitsprofils in zwei Gruppen: Die erste Gruppe war charakterisiert durch spontanes und emotional irritierbares Verhalten bei zahlreichen kriminellen Vortaten ohne Einbindung in eine Ideologie und ohne Gruppenkontakte („volatile lone actor terrorists"). Die zweite Gruppe war zwar in ein radikales oder terroristisches Milieu integriert, bevorzugte aber Terrorattacken in Eigenregie, weil sie sich hierdurch einen größeren persönlichen Erfolg versprachen („autonomous lone actors").

Der Hintergrund terroristischer Einzelattacken ist nicht an eine bestimmte Ideologie gebunden. Es findet sich bei solchen Tätern das gesamte Spektrum rechtsradikalen, linksradikalen und salafistisch-dschihadistischen Gedankengutes. Oft entwickeln solche Täter auch Hassideologien, die ihrem eigenen Gedankengut entsprungen sind und nicht einer politischen oder religiösen extremen Ideologie zugehörig sind.

Eine Gegenüberstellung von terroristischen Einzeltätern und Mitgliedern terroristischer Organisationen zeigt - Tab. 1.

In einer Studie mit 27 US-Einzeltätern aus den Jahren 1995 bis 2001 kam Hewitt zu dem Fazit, dass diese wahrscheinlicher an einer Depression oder paranoiden Symptomatik leiden als US-Terroristen, die keine Einzeltäter sind [35]. Sie leben häufiger getrennt bzw. isoliert mit nur wenigen/keinen sozialen Kontakten [16, 30, 31, 81]. 
Tab. 1 Gegenüberstellung terroristischer Einzeltäter und Gruppierungen

\begin{tabular}{|c|c|c|}
\hline Merkmal & Terroristische Einzeltäter & Terroristische Gruppierungen \\
\hline \multirow[t]{4}{*}{ Definition } & $\begin{array}{l}\text { Planung, Organisation und Ausführung ohne finanzielle/ } \\
\text { personelle Unterstützung einer terroristischen Organisation [1] }\end{array}$ & $\begin{array}{l}\text { Training, Finanzierung und Koordination durch eine terroristi- } \\
\text { sche Organisation [1] }\end{array}$ \\
\hline & Individuelle und unabhängige Operation [81] & \multirow[t]{3}{*}{ Organisatorische Struktur und finanzielle Förderung [1] } \\
\hline & Ohne Kommunikation/Interaktion mit einer Gruppe [62] & \\
\hline & Inspiration durch Internet und terroristische Gruppierungen [16] & \\
\hline \multirow[t]{3}{*}{ Mittleres Alter } & \multirow{3}{*}{$\begin{array}{l}33 \text { Jahre [30] bis Ende } 30[32] \\
\rightarrow \text { Alter von Einzeltätern höher als in vergleichbaren Studien mit } \\
\text { terroristischen Gruppierungen }\end{array}$} & Kolumbianische Kämpfer: 20 Jahre [24] \\
\hline & & Provisorische irisch-republikanische Armee (PIRA): 25 Jahre [29] \\
\hline & & Al-Qaida-Terroristen: 26 Jahre [74] \\
\hline \multirow{3}{*}{$\begin{array}{l}\text { Männliches } \\
\text { Geschlecht }\end{array}$} & \multirow[t]{3}{*}{$96,6 \%[30]$} & PIRA: 95,1\% [29] \\
\hline & & IRA: 97,3\% [27] \\
\hline & & ETA: 93,6\% [72] \\
\hline \multirow{4}{*}{$\begin{array}{l}\text { Mentale } \\
\text { Erkrankungen }\end{array}$} & $40 \%$ leiden an mentaler Erkrankung [32] & \multirow[t]{4}{*}{ 7-8\% leiden an mentaler Erkrankung [32] } \\
\hline & $\begin{array}{l}\text { 13,9-mal wahrscheinlicher psychische Erkrankung als Mitglieder } \\
\text { terroristischer Gruppierungen [16] }\end{array}$ & \\
\hline & $\begin{array}{l}61 \% \text { in der Vergangenheit Kontakt mit Dienstleistungen im } \\
\text { Bereich der psychischen Gesundheit [22] }\end{array}$ & \\
\hline & $\begin{array}{l}\rightarrow \text { Psychische Störung als möglicher Risikofaktor für terroristi- } \\
\text { sche Einzeltaten [53] }\end{array}$ & \\
\hline \multirow[t]{2}{*}{ Isolation } & $\begin{array}{l}53 \% \text { sozial isoliert [16] } \\
\rightarrow \text { Großer Anteil sozialer Isolation [30] }\end{array}$ & Organisation und Gruppendynamik [61] \\
\hline & Häufig Einzelgänger mit wenigen sozialen Kontakten [62] & Höhere Effektivität, Zugang zu finanziellen Ressourcen [1] \\
\hline \multirow[t]{2}{*}{ Vorstrafen } & \multirow[t]{2}{*}{$40[30]-50 \%[32]$ haben Vorstrafen } & Anteil an Tätern mit Vorstrafen geringer [62] \\
\hline & & Bevorzugte Rekrutierung Personen ohne Vorstrafen [30] \\
\hline \multirow[t]{3}{*}{ Letalität } & Geringere Rate an vollendeten Taten [79] & 1,60 Tote pro Attacke [79] \\
\hline & 0,62 Tote pro Attacke [79] & 2-mal so viele Todesopfer [1] \\
\hline & Kontextbedingt: Letalität signifikant erhöht in USA [63] & $\rightarrow$ Sind organisierter, ressourcenreicher und effektiver [1] \\
\hline
\end{tabular}

Corner und Gill [16] untersuchten 119 Einzeltäter-Terroristen im Vergleich $\mathrm{zu}$ einer altersangepassten Gruppe von Mitgliedern terroristischer Gruppierungen mithilfe multivariater statistischer Analysen. Sie fanden eine 13- bis 14-mal höhere Wahrscheinlichkeit (Odds Ratio) für das Vorliegen einer mentalen Erkrankung bei Einzel- im Vergleich zu Gruppentätern. Es handelt sich dabei primär um schwere psychische Erkrankungen, insbesondere Psychosen. In einer weiteren Untersuchung an 153 EinzeltäterTerroristen konnten Corner und Kollegen [17] zeigen, dass schizophrene und wahnhafte Störungen sowie Autismusspektrumstörungen in dieser Population im Vergleich zur Allgemeinbevölkerung eine höhere Prävalenz aufweisen.

Bhui und Mitarbeiter [9] haben eine Querschnittsfragebogenuntersuchung mit 608 aus Pakistan und Bangladesch gebürtigen Probanden durchgeführt. Es zeigte sich, dass depressive Symptome mit einem höheren Risiko für Sympathie bzw. Verständnis für Terrorismus assoziiert sind. Diese Autoren beschreiben darüber hinaus, dass nicht eine einzelne Diagnose für Einzeltäter-Terroristen typisch ist, sondern ein breites Spektrum von Persönlichkeitsstörungen, schizophrenen Psychosen, anhaltenden wahnhaften Störungen oder Autismusspektrumstörungen vorliegt. Posttraumatische Belastungsstörungen, Angst, Depression sowie Alkoholabhängigkeit werden laut einer anderen Übersichtsarbeit [2] ebenfalls bei Einzeltätern gehäuft angetroffen.

Es gibt Berichte, dass terroristische Organisationen sich von offensichtlich gestörten Menschen distanzieren (z.B. $[23,34])$, da das Einbeziehen solcher Mitglieder die terroristische Effektivität negativ beeinflussen könnte [74].

\section{Rechtsterrorismus und Verschwörungstheorien}

Verschwörungstheorien bilden den ideologischen Kern vor allem rechten Terrors. Die Täter der Anschläge von Utoya, München, Christchurch, Poway, El-Paso, Baerum, Halle (Saale) sowie Hanau beriefen sich auf verschiedene Variationen der Weltverschwörungstheorie des Great Replacement, White Genocide oder Eurobia, welche größtenteils inhaltliche Überschneidungen aufweisen [19]. Kern dieser realitätsfremden Theorien ist, dass die weiße europäische Bevölkerung bewusst reduziert und letztlich durch andere ethnische/religiöse Gruppen (z. B. Muslime) ersetzt wird, deren Kultur mit der westlichen unvereinbar sei [19]. Diese Narrative vereint verschiedenste rechtextremistische, antifeministische, antisemitische, Antiestablishment- und Antimigrationsideologien zu einer. In Deutschland auch als „Großer Austausch“ oder „Volkstod“ 
bezeichnet war ähnliches Gedankengut bereits in „Mein Kampf“"vorhanden [4].

Nach Imhoff und Lamberty [37] weisen Paranoia und Verschwörungsdenken eine gewisse Verwandtschaft auf und zeigen eine schwache Korrelation in psychologischen Bewertungsskalen auf, sind aber keine identischen Phänomene.

Paranoid-psychotische Symptome sind immer selbstreferenziell; die vermeintliche Bedrohung oder Beeinflussung wird von den betroffenen Menschen auf sich selbst gerichtet empfunden. Bei Verschwörungstheorien liegt hingegen von vornherein eine feindselige Einstellung gegenüber anderen Menschengruppen vor. Die wahrgenommene Bedrohung, wenn auch realitätsfremd, wird auf die Gesellschaft oder eigene Gruppen und nicht nur auf sich selbst bezogen. Eine psychotische Symptomatik muss nicht vorliegen, kann aber auch bei einzelnen Tätern mit Verschwörungstheorien zusammen auftreten, wie z. B. bei dem sog. Unabomber, der in den USA zwischen 1978 und 1995 durch Briefbomben drei Menschen tötete und 16 verletzte [49], und auch bei dem Täter von Hanau [60]. Die realitätsgestörte Einordnung vermeintlicher Feinde gegen die Eigengruppe fungiert als kognitiver Hauptmechanismus des Verschwörungsglaubens [68] und dient als Rechtfertigung für Terrorhandlungen. Dadurch soll die nicht vorhandene soziale oder politische Kontrolle über die anderen kompensiert werden [71]. Hinzu kommen archaische soziale Motive von Verschwörungstheorien. Sie liefern Narrative zur Abwertung der Fremdgruppe und Aufwertung der Eigengruppe, welche keine objektiven Wahrheiten benötigen und deshalb besonders ansprechend für Randgruppen sind [20].

\section{Terrorismus als vorwiegend männliches Phänomen}

Die Mehrzahl von Terrorakten - wie individuelle und kollektive Gewalttaten überhaupt - wird durch junge Männer ausgeübt $[58,59]$. In einigen Terrorgruppierungen spielten jedoch auch Terroristinnen eine wesentliche Rolle (z. B. RAF: Ulrike Meinhof, NSU: Beate Zschäpe).
Im Gegensatz zu Einzeltätern bilden terroristische Gruppierungen eine kollektive Identität, die geprägt ist durch eine sog. Ingroup-outgroup-Feindschaft. Gruppenprozesse sowie ein charismatischer Anführer, dem die Mitglieder gehorsam sind, werden hierbei als wichtige Faktoren angesehen [5, 67].

Extremisten teilen, unabhängig von ihrer ideologischen Ausrichtung (ob rechtsextrem oder islamistisch), die Einstellung, dass ihre fundamentalistischen, politischen oder religiösen Werte durch westliche Modernisierungsprozesse bedroht sind, was auch für die traditionelle patriarchale Geschlechterordnung zutrifft. Daher ist es nicht verwunderlich, dass diese Gruppen in ihren Botschaften, Videos und Symbolen ein ultrakonservatives Männlichkeitsideal propagieren, das als Voraussetzung für die Schaffung einer besseren Welt gesehen wird [80].

Als eine von vielen gesellschaftlichen Ursachen für Gewalt und Terrorismus wird der demographische Überschuss junger Männern im Alter von 15 bis 24 Jahren diskutiert. „More men, more violence?“ [75]. Ein solcher „youth bulge“ von etwa $20 \%$ ist besonders in den islamischen Staaten ausgeprägt und soll nach Heinsohn [34] die Hauptursache für die Entwicklung und Eskalation des Terrors sein, da der Jungmännerüberschuss ein unerschöpfliches Reservoir für die Rekrutierung von Terroristen darstelle. Der zentrale Grund wird darin gesehen, dass junge Männer in diesen Ländern (z.B. Nordafrika, Mittlerer Osten, Teile von Asien) angesichts einer stagnierenden wirtschaftlichen Entwicklung geringe Aussichten auf Erwerbstätigkeit, Partnerschaft und Familiengründung haben und sich alternativ in gewaltsamen Konflikten engagieren. Dies gilt nicht nur für desintegrierte junge Männer mit wenig Bildung, sondern auch für hoch qualifizierte, für die keine adäquaten Positionen zur Verfügung stehen [26]. So sind z. B. junge Ingenieure unter den islamistischen Terroristen in muslimischen Ländern überrepräsentiert. Allerdings lässt sich anhand historischer Daten und einschlägiger Studien ein monokausaler, linearer Zusammenhang zwischen Männerüberschuss und Terrorismus eben so wenig belegen, wie zwischen Armut und
Terrorismus [54, 75]. Die simple Annahme "more men, more violence" [75] übersieht die Bedeutung der sozioökonomischen Struktur einer Gesellschaft, die jungen statusorientierten Männern Chancen auf Zukunft bieten muss.

Ein Überschuss an Männern ist nicht nur in muslimischen Ländern, in Indien und China ein Problem, sondern auch in Teilen von Deutschland. So hat sich etwa in Ostdeutschland seit der Wiedervereinigung 1989 aufgrund der im Vergleich zu Westdeutschland geringeren wirtschaftlichen Entwicklung und der Abwanderung gut ausgebildeter junger Frauen in einigen ländlichen Regionen ein Überschuss an jungen Männern gebildet, deren Situation durch mangelnde Bildung, Arbeits- und Partnerlosigkeit, Perspektivlosigkeit und Frustration gekennzeichnet ist. Unter diesen Bedingungen ist die Zahl der Rechtsextremisten im Zeitraum 2014 bis 2018 von 21.000 auf 24.100 gestiegen [13, 15], wobei die Hälfte als gewaltorientiert eingeschätzt werden muss [42]. Von 2014 bis 2016 stieg die Zahl der Gewalttaten um $72 \%$ $[13,14]$, wobei es sich überwiegend um Angriffe auf Flüchtlingsunterkünfte handelte. Im Anschluss sank die Anzahl der Gewalttaten wieder, dennoch ist im Zeitraum von 2014 bis 2018 ein Anstieg von $10 \% \mathrm{zu}$ verzeichnen $[13,15]$.

\section{Gruppendynamik und kollektive Identität}

Grundsätzlich stellen homosoziale Gruppen Gleichaltriger eine wesentliche Instanz der männlichen Sozialisation im Jugendalter dar, die Bedürfnisse nach Zugehörigkeit und Anerkennung, sozialer Identität, Status, Wettbewerb und Demonstration von Maskulinität erfüllen. So ist es nicht verwunderlich, dass $90 \%$ aller Gewalttaten von jungen Männern in Gruppen ausgeübt werden [87] und Terroristen sich oft in Gruppen organisieren. Experimentelle Studien belegen, dass Gruppen im Vergleich $\mathrm{zu}$ Individuen aggressiver und kompetitiver sind [55], ein Befund, der kompatibel mit evolutionsbiologischen Ansätzen ist, die davon ausgehen, dass Gruppenbildung Überlebensvorteile sicherte und begrenzte Ressourcen mit 
Aggression und Gewalt effektiver gegenüber rivalisierenden Gruppen verteidigt werden konnte. Das von der Sozialpsychologie beschriebene Phänomen der Intergruppendiskriminierung, das sich mit zahllosen Kriegen, Kämpfen und Genoziden durch die Weltgeschichte zieht, beruht auf der Überbewertung der eigenen (,ingroup“) und der Abwertung der fremden Gruppe („outgroup“), wobei die Gruppenunterschiede völlig beliebig und minimal sein können („minimal group paradigm“ [84]), aber Diskriminierung und Bekämpfung legitimieren.

Kollektive Identität stärkt sowohl das Selbstwertgefühl des Einzelnen als auch die Gruppenkohäsion. Terroristische Gruppen weisen aufgrund ihrer ideologischen Mission eine besonders hohe Gruppenkohäsion auf und sind damit hoch attraktiv für potenzielle Terroristen, die weniger an der Ideologie als an Freundschaft und Gemeinschaft interessiert sind [77].

\section{Rolle der Psychiatrie in der Prävention}

In ihrer Analyse der soziodemographischen Netzwerke von insgesamt 119 Einzeltat-Terroristen beschreiben Gill und Kollegen, dass häufig die Personen im Umfeld extremistischer Ideologien von der Absicht Kenntnis hatten, dass Terrorakte ausgeübt werden sollten, die zudem lange und intensiv vorbereitet wurden [30]. Allerdings ist es wenig wahrscheinlich, dass Psychiater oder Psychotherapeuten hierüber Kenntnis aus dem Umfeld von Terrorgruppen bekommen. Erfolgversprechender scheint bei Kenntnis des Risikoprofils die Früherkennung terroristischer Einzeltäter zu sein.

Es gibt verschiedene Risikobewertungsinstrumente, die der Einschätzung der Wahrscheinlichkeit extremistischer Gewalttaten dienen. Dazu zählen das „Violent Extremism Risk Assessment“ (Version 2 Revised, VERA-2R; [73]), das „Terrorist Radicalization Assessment Protocol“ (TRAP-18; [56]) sowie die vom BKA und der Universität Konstanz entwickelte „Regelbasierte Analyse potenziell destruktiver Täter zur Einschätzung des akuten Risikos islamistischen Terrorismus“ (RADAR-iTE; [12]). Ein neues Modellprojekt, was sich spezifisch an rechtsextrem orientierte junge Menschen richtet, ist "Online Hass Abbauen“ (OHA; [21]). Hierbei handelt es sich um ein virtuelles Training zur Reduktion von Hass und Gewalt. Ein vom BMBF gefördertes, groß angelegtes Projekt ist „Radikalisierung im digitalen Zeitalter - Risiken, Verläufe und Strategien der Prävention“ (RadigZ; [43]). Im Fokus dieses Verbundprojekts stehen unter anderem die Ermittlung von Vulnerabilitätsfaktoren und die Entwicklung von Präventionsmaßnahmen.

\section{Schlussfolgerung}

Wenn man von forensischen Fragestellungen absieht, zeigt die Psychiatrie bislang kaum Interesse für das multidimensionale Bedingungsgefüge von Gewalt; das trifft auch für die Ursachen von Terrorhandlungen Einzelner oder durch Gruppen zu. Dieses Feld wurde weitgehend Sozialwissenschaftlern, Kriminologen, Psychologen und auch Politikern überlassen. Terrorhandlungen sind aber das Resultat elementarer Emotionen, wie Hass, Fanatismus, Aggressivität oder auch Geltungsbedürfnis und Überlegenheitsdenken, für die es ebenso wie für andere Emotionen, mit denen sich die Psychiatrie hinreichend beschäftigt (wie z.B. Angst, Depression und Euphorie/Manie), einen breiten Übergangsbereich zwischen Normalpsychologie und Psychopathologie gibt. Mitunter scheinen Terrorhandlungen auch einem reinen Vernichtungswillen Andersartiger zu entspringen, der kaum aus einem bekannten Psychosyndrom herzuleiten ist.

Bei einem hohen Anteil terroristischer Einzeltäter, die überwiegend sozial isoliert leben, lassen sich psychische Störungen der Achse I nach DSM-IV, überwiegend psychotische, wahnhafte aber auch posttraumatische und Autismusspektrumstörungen, feststellen. Dahingegen konnten bei Mitgliedern von Terrorgruppen nur in seltenen Einzelfällen derartige psychische Auffälligkeiten von Krankheitswert festgestellt werden. Es wurden eher Persönlichkeitsakzentuierungen bis hin zu Persönlichkeitsstörungen antisozialer und histrionischer
Art, somit Syndrome der Achse II nach DSM-IV, beschrieben.

Das Vulnerabilitäts-Stress-Konzept, wonach die Vulnerabilität durch die vorbestehende (biografisch, genetisch, neurobiologisch geprägte) Persönlichkeitskonfiguration gegeben ist und die Stresskomponente durch die aktuelle psychosoziale und politische Situation, verstärkt durch gruppendynamische Einbindung, hat auch für das Phänomen Terror einen hohen Erklärungswert. Danach können auch bei sehr geringer Vulnerabilität stärkste Stressoren Terrorhandlungen auslösen und umgekehrt bei ausgeprägter Persönlichkeitsdisposition $\mathrm{zu}$ Gewalthandlungen geringe Anlässe hierfür ausreichen.

Die politischen, religiösen, rassistischen oder sonstigen Ideologien, in deren Namen Terror verübt wird, sind das ist eine wesentliche Schlussfolgerung der Autoren - nicht selbst der Grund für terroristische Gewalttaten, sondern werden von gewaltbereiten Individuen aktiv aufgesucht, um dadurch ein Gefühl von Zurücksetzung auszugleichen sowie Überlegenheit und Bedeutsamkeit oder einfach nur eine Befriedigung durch die Terrorhandlung zu erlangen. Solche Täter im Vorfeld zu erkennen und damit prophylaktisch aktiv zu werden, ist naturgemäß äußerst schwierig, auch deshalb, weil terroristische Einzeltäter mit psychischen Störungen kaum soziale Kontakte haben. Die Psychiatrie sollte aber von den in den letzten Jahren entwickelten Risikobewertungsinstrumenten Kenntnis nehmen, um gegebenenfalls therapeutische Maßnahmen einleiten zu können, auch wenn dies nur in wenigen Einzelfällen möglich sein dürfte.

\section{Fazit für die Praxis}

\footnotetext{
- Entgegen der landläufigen Meinung weisen Mitglieder von Terrorgruppen kein erhöhtes Vorkommen krankheitswertiger psychischer Störungen auf. Stattdessen finden sich hier gehäuft Persönlichkeitsakzentuierungen histrionischer, antisozialer und paranoid-aggressiver Art sowie eine Disposition zu gewalttätigem Verhalten, das durch soziale und
} 
gruppendynamische Gegebenheiten hergeleitet werden kann.

- Terroristische Einzeltäter leiden hingegen gehäuft an psychotischen oder affektiven Störungen. Die radikale Entwicklung solcher Täter entspringt entweder eigenem (paranoidem) Gedankengut oder erfolgt bei digitaler Vernetzung mit Gleichgesinnten über Hassforen im Internet.

- Verschwörungstheorien unterscheiden sich von paranoiden Syndromen dadurch, dass sie nicht von Einzelpersonen auf sich selbst bezogen werden, sondern sich eine Gruppe ohne realen Anlass durch eine vermeintlich feindliche andere Gruppe bedroht sieht.

- Traditionelle Ideale von Maskulinität und archaische Vorstellungen von Kampf und Heldentum tragen zur Erklärung bei, dass Terrorismus ein überwiegend männliches Phänomen ist.

- Mehrere in den letzten Jahren entwickelte Skalen können zur Früherkennung eines Risikos für extreme Gewalttaten beitragen.

\section{Korrespondenzadresse}

Prof. Dr. med. B. Bogerts

Salus Institut, Salus gGmbH

Seepark 5, 39116 Magdeburg, Deutschland b.bogerts@salus-Isa.de

Funding. Open Access funding provided by Projekt DEAL.

\section{Einhaltung ethischer Richtlinien}

Interessenkonflikt. S. Seidenbecher, C. Steinmetz, A.-M. Möller-Leimkühler und B. Bogerts geben an, dass kein Interessenkonflikt besteht.

Für diesen Beitrag wurden von den Autoren keine Studien an Menschen oder Tieren durchgeführt. Für die aufgeführten Studien gelten die jeweils dort angegebenen ethischen Richtlinien.

Open Access. Dieser Artikel wird unter der Creative Commons Namensnennung 4.0 International Lizenz veröffentlicht, welche die Nutzung, Vervielfältigung, Bearbeitung, Verbreitung und Wiedergabe in jeglichem Medium und Format erlaubt, sofern Sie den/die ursprünglichen Autor(en) und die Quelle ordnungsgemäß nennen, einen Link zur Creative Commons Lizenz beifügen und angeben, ob Änderungen vorgenommen wurden.
Die in diesem Artikel enthaltenen Bilder und sonstiges Drittmaterial unterliegen ebenfalls der genannten Creative Commons Lizenz, sofern sich aus der Abbildungslegende nichts anderes ergibt. Sofern das betreffende Material nicht unter der genannten Creative Commons Lizenz steht und die betreffende Handlung nicht nach gesetzlichen Vorschriften erlaubt ist, ist für die oben aufgeführten Weiterverwendungen des Materials die Einwilligung des jeweiligen Rechteinhabers einzuholen.

Weitere Details zur Lizenz entnehmen Sie bitte der Lizenzinformation auf http://creativecommons.org/ licenses/by/4.0/deed.de.

\section{Literatur}

1. Alakoc BP (2015) Competing to kill: Terrorist organizations versus lone wolf terrorists. Terror Polit Violence 29:1-24. https://doi.org/10.1080/ 09546553.2015.1050489

2. Alderdice TL (2007) The individual, the group and the psychology of terrorism. Int Rev Psychiatry 19:201-209. https://doi.org/10.1080/ 09540260701346825

3. Alonso J, Angermeyer MC, Bernert S et al (2004) Prevalence of mental disorders in Europe: results from the European Study of the Epidemiology of Mental Disorders (ESEMeD) project. Acta Psychiatr Scand 109:21-27. https://doi.org/10.1111/j.1600 0047.2004.00327.x

4. Amadeu Antonio Stiftung (2019) Wissen was wirklich gespielt wird, Widerlegung für gängige Verschwörungstheorien. https://www.amadeuantonio-stiftung.de/publikationen/wissen-waswirklich-gespielt-wird/. Zugegriffen: 25. Febr. 2020

5. Atran S (2016) The devoted actor: unconditional commitment and intractable conflict across cultures. Curr Anthropol 57:S192-S203. https:// doi.org/10.1086/685495

6. Bartlett J, Miller C (2012) The edge of violence: towards telling the difference between violent and non-violent radicalization. Terror Polit Violence 24:1-21. https://doi.org/10.1080/09546553.2011. 594923

7. Bhui K, Everitt B, Jones E (2014) Might depression, psychosocial adversity, and limited social assets explain vulnerability to and resistance against violent radicalisation? Plos One 9:e105918. https:// doi.org/10.1371/journal.pone.0105918

8. Bhui K, James A, Wessely S (2016) Mental illness and terrorism. BMJ. https://doi.org/10.1136/bmj. i4869

9. BhuiK, Silva MJ, Topciu RA, Jones E (2016) Pathways to sympathies for violent protest and terrorism. $\mathrm{Br} J$ Psychiatry 209:483-490. https://doi.org/10.1192/ bjp.bp.116.185173

10. Bogerts B, Peter E, Schiltz K (2011) Aggression, Gewalt, Amok, Stalking. In: Möller H-J, Laux G, Kapfhammer H-P (Hrsg) Psychiatrie, Psychosomatik, Psychotherapie. Springer, Berlin, Heidelberg, $S$ 2692-2716

11. Bundeskriminalamt (2016) Analyse der den deutschen Sicherheitsbehörden vorliegenden Informationen über die Radikalisierungshintergründe und -verläufe der Personen, die aus islamistischer Motivation aus Deutschland in Richtung Syrien ausgereist sind. https://www.bka. de/SharedDocs/Downloads/DE/Publikationen/ Publikationsreihen/Forschungsergebnisse/ 2016AnalyseRadikalisierungsgruendeSyrienlrak Ausreisende.html.Zugegriffen:25. Febr. 2020
12. Bundeskriminalamt (2017) Presseinformation: Neues Instrument zur Risikobewertung von potentiellen Gewaltstraftätern. bka.de/DE/ Presse/Listenseite_Pressemitteilungen/2017/ Presse2017/170202_Radar.html. Zugegriffen: 18. Dez. 2019

13. Bundesministerium des Innern für Bau und Heimat (2015) Verfassungsschutzbericht 2014

14. Bundesministerium des Innern für Bau und Heimat (2017) Verfassungsschutzbericht 2016. https://www.verfassungsschutz. de/de/oeffentlichkeitsarbeit/publikationen/ verfassungsschutzberichte/vsbericht-2016. Zugegriffen: 25. Febr. 2020

15. Bundesministerium des Innern für Bau und Heimat (2019) Verfassungsschutzbericht 2018. https://www.verfassungsschutz. de/de/oeffentlichkeitsarbeit/publikationen/ verfassungsschutzberichte/vsbericht- 2018 Zugegriffen: 25. Febr. 2020

16. Corner E, Gill P (2015) A false dichotomy? Mental illness and lone-actor terrorism. Law Hum Behav 39:23-34.https://doi.org/10.1037//hb0000102

17. Corner E, Gill P, Mason O (2016) Mental health disorders and the terrorist: a research note probing selection effects and disorder prevalence. Stud Confl Terror 39:560-568. https://doi.org/10.1080/ 1057610X.2015.1120099

18. Crenshaw M (1986) The psychology of poltical terrorism. In: Hermann MG (Hrsg) Political psychology. Contemporary problems and issues. Jossey-Bass, San Francisco, S 379-413

19. Davey J, Ebner J (2019) The great replacement, the violent consequences of mainstreamed extremism. Institute for Strategic Dialogue, London, Großbritannien, S1-36

20. Douglas KM, Douglas KM, Sutton RM (2017) The psychology of conspiracy theories. Curr Dir Psychol Sci.https://doi.org/10.1177/0963721417718261

21. Drudel 11 e. V. OHA Online Hass abbauen. http:// blog.oha-training.de.Zugegriffen:25. Febr. 2020

22. Fein R, Vossekuil B (1999) Assassination in the United States: an operational study of recent assassins, attackers, and near-lethal approachers. JForensic Sci 44:321-333

23. Fetscher C (2018) Gegen Kapitalismus und Irrenhaus. https://www.tagesspiegel.de/kultur/ spkkomplex-im-forum-gegen-kapitalismusund-irrenhaus/20988368.html. Zugegriffen: 1. Okt. 2018

24. Florez-Morris M (2007) Joining guerrilla groups in Colombia: individual motivations and processes for entering a violent organization. Stud Confl Terror 30:615-634. https://doi.org/10.1080/ 10576100701385958

25. Fredholm M (2011) Hunting lone wolves - finding Islamist lone actors before they strike. Stockholm seminar on lone wolf terrorism

26. Gambetta D, Hertog S (2016) Engineers of jihad: the curious connection between violent extremism and education. Princeton Univers. Press, Princeton

27. Gill P (2011) Who becomes a dissident? Patterns in the mobilisation and recruitment of violent dissident republicans in northern Ireland. In: Taylor M, Currie PM (Hrsg) Dissident Irish republic, S43-64

28. Gill P, Corner E (2017) There and back again: The study of mental disorder and terrorist involvement. Am Psychol 72:231-241. https://doi.org/10.1037/ amp0000090

29. GillP,Horgan J (2013) Who were the volunteers?The shifting sociological and operational profile of 1240 provisional Irish republican army members. 
Terror Polit Violence 25:435-456. https://doi.org/ 10.1080/09546553.2012.664587

30. Gill P, Horgan J, Deckert P (2014) Bombing alone: Tracing the motivations and antecedent behaviors of lone-actor terrorists. J Forensic Sci 59:425-435. https://doi.org/10.1111/1556-4029.12312

31. Gruenewald J, Chermak S, Freilich JD (2013) Distinguishing "Loner" attacks from other domestic extremist violence. Criminol Public Policy 12:65-91. https://doi.org/10.1111/1745-9133. 12008

32. Gruenewald J, Chermak S, Freilich JD (2013) Farright lone wolf homicides in the United States. Stud Confl Terror 36:1005-1024. https://doi.org/ 10.1080/1057610X.2013.842123

33. Hare RD (1991) The hare psychopathy checklistrevised $(\mathrm{PCL}-\mathrm{R})$. Multi-Health Systems, Toronto, Ontario

34. Heinsohn G (2006) Söhne und Weltmacht. Terror im Aufstieg und Fall der Nationen. Orell Füssli, Zürich

35. Hewitt C (2003) Understanding terrorism in America. Routledge, New York

36. Horgan J (2014) The psychology of terrorism, 2. Aufl. Routledge, New York

37. Imhoff R, Lamberty P (2018) How paranoid are conspiracy believers? Toward a more fine-grained understanding of the connect and disconnect between paranoia and beliefin conspiracy theories connect and disconnect between paranoia and belief in conspiracy theories. Eur J Soc Psychol. https://doi.org/10.1002/ejsp.2494

38. Institute for Economics \& Peace (2019) Global terrorism index 2019. Measuring the impact of terrorism

39. Jäger H, Böllinger $L$ (1981) Thesen zur weiteren Diskussion des Terrorismus. In: Jäger $\mathrm{H}$, Schmidtchen G, Süllwold L (Hrsg) Lebenslaufanalysen. VS, Wiesbaden, S231-236

40. Jense M, James P, Lafree $G$ et al (2018) The use of social media by United States extremists. START, College Park

41. Jensen MA, Atwell Seate A, James PA (2018) Radicalization to violence: a pathway approach to studying extremism. Terror Polit Violence. https:// doi.org/10.1080/09546553.2018.1442330

42. Koehler D (2016) Right-wing extremism and terrorism in Europe. Current developments and issues for the future. Prism 6:84-105. https://doi. org/10.2190/CS.16.1.d

43. Kudlacek D, Jukschat N, Beelmann A et al (2017) Radikalisierung im digitalen Zeitalter - Risiken, Verläufe und Strategien der Prävention. Forum Kriminalprävention 3:23-32

44. Kumpfmüller K (2019) Online Subkultur des Hasses. https://www.tagesschau.de/faktenfinder/ online-subkultur-103.html. Zugegriffen: 25 . Febr. 2020

45. Lauritsen JL, Heimer K, Lynch JP (2009) Trends in the gender gap in violent offending: new evidence from the national crime victimization survey. Criminology 47:361-399. https://doi.org/ 10.1111/j.1745-9125.2009.00149.x

46. LeuschnerV,BöcklerN,ZickA,ScheithauerH(2017) Attentate durch Einzeltäter. Zu Gemeinsamkeiten in der Tatentwicklung und der Tatsituation bei terroristischen Anschlägen und School Shootings. In: Böckler N, Hoffmann J (Hrsg) Radikalisierung und Terror. Gewalt. Perspekt. aus dem Fall- und Bedrohungsmanagement. Verlag für Polizeiwissenschaft, Frankfurt, S51-78

47. LeygrafN (2014)ZurPhänomenologie islamistischterroristischer Straftäter. Forens Psychiatr Psychol
Kriminol 8:237-245. https://doi.org/10.1007/ s11757-014-0291-2

48. Lindekilde L, O'Connor F, Schuurman B (2019) Radicalization patterns and modes of attack planning and preparation among lone-actor terrorists: an exploratory analysis. Behav Sci Terro Polit Aggress 11:113-133. https://doi.org/10. 1080/19434472.2017.1407814

49. Lörchner J (2018) ,Unabomber' Ted Kaczynski: Der Briefbomben-Eremit. https://www.spiegel. de/geschichte/unabomber-theodore-tedkaczynski-der-briefbomben-eremit-a- 1204440. html.Zugegriffen:25. Febr. 2020

50. Maier W, Hauth I, Berger M, SaßH (2016) Zwischenmenschliche Gewalt im Kontext affektiver und psychotischer Störungen. Nervenarzt 87:53-68

51. Mania-Schlegel J (2019) Ich bereue es, 8chan gegründet zu haben. https://www.zeit.de/digital/ internet/2019-10/frederick-brennan-8changruender-attentat-halle-radikalisierung. Zugegriffen:25. Febr. 2020

52. Marazziti D, Stahl SM (2018) Evil, terrorism, and psychiatry. CNS Spectr 23:117-118. https://doi. org/10.1017/S1092852917000517

53. McCauley C, Moskalenko S, Van Son B (2013) Characteristics of the lone-wolf violent offenders: a comparison of assassins and school attackers. Perspect Terror 7:3-22

54. McGilloway A, Ghosh P, Bhui K (2015) A systematic review of pathways to and processes associated with radicalization and extremism amongst Muslims in Western societies. Int Rev Psychiatry 27:39-50. https://doi.org/10.3109/09540261. 2014.992008

55. Meier BP, Hinsz VB (2004) A comparison of human aggression committed by groups and individuals: an interindividual-intergroup discontinuity. J Exp Soc Psychol 40:551-559. https://doi.org/10.1016/ j.jesp.2003.11.002

56. Meloy JR, Gill P (2016) The lone-actor terrorist and the TRAP-18. J Threat Assess Manag 3:37-52. https://doi.org/10.1037/tam0000061

57. Merari A (2010) Driven to death. Psychological and social aspects of suicide terrorism. Oxford University Press, Oxford

58. Möller-Leimkühler AM (2017) Why is terrorism a man's business? CNS Spectr. https://doi.org/10. 1017/S1092852917000438

59. Möller-Leimkühler AM, Bogerts B (2013) Kollektive Gewalt. Nervenarzt 84:1345-1358. https://doi. org/10.1007/s00115-013-3856-y

60. Musharbash Y, Sundermann T (2020) Täter von Hanau: Rassismus, Verschwörung und Paranoia. https://www.zeit.de/ gesellschaft/zeitgeschehen/2020-02/taeterhanau-rassismus-rechtsextremismus-motivschreiben.Zugegriffen:24. Febr. 2020

61. Nesser P (2012) Research notes and resources research note: single actor terrorism: scope. Charact Explan 6:61-73

62. Pantucci R, Ellis C, Chaplais L (2015) Lone-actor terrorism. Literature review. Royal United Service Institute, London

63. Phillips BJ (2017) Deadlier in the U.S.? On lone wolves, terrorist groups, and attack lethality. Terro Polit Violence 29:533-549. https://doi.org/10. 1080/09546553.2015.1054927

64. Piccinni A, Marazziti D, Veltri A (2018) Psychopathology of terrorists. CNS Spectr 23:141-144. https:// doi.org/10.1017/S1092852917000645

65. Post JM (1998) Terrorist psycho-logic: terrorist behaviour as a product of psychological forces. In: Reich W, LaqueurW (Hrsg) Origins of terrorism: psychologies, ideologies, theologies, states of mind.
Woodrow Wilson Center, Baltimore, Washington, $\mathrm{S}$ 25-42

66. Post JM (2008) The mind of the terrorist: the psychology of terrorism from the IRA to al-Qaeda, 1. Aufl. Martin's Griffin, New York

67. Post JM, McGinnis C, Moody K (2014) The changing face of terrorism in the 21st century: the communications revolution and the virtual community of hatred. Behav Sci Law 32:306-334. https://doi.org/10.1002/bsl.2123

68. Van Prooijen J, Douglas KM, De Inocencio C (2018) Connecting the dots: Illusory pattern perception predicts belief in conspiracies and the supernatural. Eur J Soc Psychol 48:320-335. https://doi.org/10.1002/ejsp.2331

69. Rapoport DC (2002) The four waves of rebel terror and September 11. Anthropoetics 8 (1)

70. Rasch W (1979) Psychological dimensions of political terrorism in the Federal Republic of Germany. Int J Law Psychiatry 2:79-85. https://doi. org/10.1016/0160-2527(79)90031-1

71. Rees JH, Lamberty P (2019) Mitreißende Wahrheiten, Verschwörungsmythen als Gefahr für den gesellschaftlichen Zusammenhalt. In: Verlorene Mitte Feindselige Zustände, Rechtsextreme Einstellungen Deutschland 2018/2019, 1. Aufl. Friedrich-Ebert-Stiftung, Bonn, S203-221

72. ReinaresF (2004) Who are the terrorists? Analyzing changes in sociological profile among members of ETA. Stud Confl Terror 27:465-488. https://doi.org/ 10.1080/10576100490519741

73. Sadowski F, Rossegger A, Pressman E et al (2017) Das Violent Extremism Risk Assessment Version 2 Revised (VERA-2R): eine Skala zur Beurteilung des Risikos extremistischer Gewalt. Kriminalstatistik 5:335-342 (Deutsche Übersetzung)

74. Sageman M(2004) Understanding terror networks. University of Pennsylvania Press, Philadelphia

75. Schacht R, Rauch KL, Borgerhoff Mulder M (2014) Too many men: the violence problem? Trends Ecol Evol 29:214-222. https://doi.org/10.1016/j.tree. 2014.02.001

76. Schmidtchen G (1981) Terroristische Karrieren: Soziologische Analyse anhand von Fahndungsunterlagen und Prozeßakten. In: Jäger $\mathrm{H}$, Schmidtchen G, Süllwold L (Hrsg) Lebenslaufanalysen. VS, Wiesbaden, S13-77

77. Simi P, WindischS, SporerK(2016) Recruitment and radicalization among US far right terrorists. START, College Park

78. Simon JD (2013) Lone wolf terrorism. Prometheus Books, Amherst

79. Smith BL, Gruenewald J, Roberts P, Damphousse KR (2015) The emergence of lone wolf terrorism: patterns of behavior and implications for intervention. In:Deflem M(Hrsg) Terrorismand counterterrorism today. Emerald Group, Bingley, S89-110

80. Society TSA for $Y$ and C (2016) Young and extreme-a youth and gender perspective on violent extremism

81. Spaaij R (2012) Understanding lone wolf terrorism: global patterns, motivations, and prevention. Springer, New York

82. Der Spiegel (2020) Irre gefährlich. Der Spiegel, S 10-18 (H9,74 Jahrgang)

83. Süllwold L (1981) Stationen in der Entwicklung von Terroristen: Psychologische Aspekte biografischer Daten.In:Jäger H,Schmidtchen G, Süllwold L(Hrsg) Lebenslaufanalysen. VS, Wiesbaden, S79-116

84. Tajfel H, Turner J (2001) An integrative theory of intergroup conflict. In: Hogg MA, Abrams D (Hrsg) Intergroup relations. Psychology Press, New York, S94-109 


\section{Übersichten}

85. Taylor M, Ryan H (1988) Fanaticism, political suicide and terrorism. Terrorism 11:91-111. https://doi. org/10.1080/10576108808435703

86. Webber D, Kruglanski AW (2018) The social psychological makings of a terrorist. Curr Opin Psychol 19:131-134. https://doi.org/10.1016/j. copsyc.2017.03.024

87. Zwiener U, Kodalle KM (2003) Gewalt und Extremismus in der heutigen Gesellschaft. Über Hintergründe, Vorbeugung und Therapie -

eine Einführung. Extrem. - Gewalt - Terror.

Hintergründe und Handlungskonsequenzen. Coll.

Eur.Jenense, Jena, Erlangen, Deutschland

Hier steht eine Anzeige.

Springer 